\title{
Electronic Properties and Density of States of Self-Assembled GaSb/GaAs Quantum Dots
}

\author{
T. Nowozin, ${ }^{1}$ A. Wiengarten, ${ }^{1}$ L. Bonato, ${ }^{1}$ D. Bimberg, ${ }^{1,2}$ Wei-Hsun Lin, ${ }^{3}$ Shih-Yen Lin, ${ }^{3}$ \\ M. N. Ajour, ${ }^{4}$ K. Daqrouq, ${ }^{4}$ and A. S. Balamesh ${ }^{4}$ \\ ${ }^{1}$ Institut für Festkörperphysik, Technische Universität Berlin, Hardenbergstraße 36, 10623 Berlin, Germany \\ ${ }^{2}$ King Abdulaziz University, Jeddah 21589, Saudi Arabia \\ ${ }^{3}$ Research Center for Applied Sciences, Academia Sinica, Taipei 11529, Taiwan \\ ${ }^{4}$ Electric and Computer Engineering Department, King Abdulaziz University, Jeddah 21589, Saudi Arabia
}

Correspondence should be addressed to T. Nowozin; nowozin@sol.physik.tu-berlin.de

Received 1 July 2013; Accepted 30 July 2013

Academic Editor: Xiao Wei Sun

Copyright (C) 2013 T. Nowozin et al. This is an open access article distributed under the Creative Commons Attribution License, which permits unrestricted use, distribution, and reproduction in any medium, provided the original work is properly cited.

\begin{abstract}
The electronic properties of a self-assembled GaSb/GaAs QD ensemble are determined by capacitance-voltage $(C-V)$ and deep-level transient spectroscopy (DLTS). The charging and discharging bias regions of the QDs are determined for different temperatures. With a value of $335( \pm 15) \mathrm{meV}$ the localization energy is rather small compared to values previously determined for the same material system. Similarly, a very small apparent capture cross section is measured $\left(1 \cdot 10^{-16} \mathrm{~cm}^{2}\right)$. DLTS signal analysis yields an equivalent to the ensemble density of states for the individual energies as well as the density function of the confinement energies of the QDs in the ensemble.
\end{abstract}

\section{Introduction}

$\mathrm{GaSb} / \mathrm{GaAs}$ quantum dots are an interesting material system due to their type-II band alignment with its exclusive hole confinement and a barrier present for electrons [1]. In particular for charge storage applications, they are a promising option due to not only the very large barriers that can be achieved [2-5] but also the spatial separation of electrons and holes which facilitates long exciton lifetimes $[6,7]$ and could lead to interesting long-wavelength optoelectronic applications [8]. Although growth by metal organic vapor phase epitaxy (MOCVD) has been demonstrated $[9,10]$, the common way to fabricate these dots is molecular beam epitaxy (MBE) due to the peculiarities of Sb (i.e., smaller vapor pressure of $\mathrm{Sb}$ as compared to $\mathrm{As}, \mathrm{Sb}$ crystal formation). For the use of GaSb/GaAs QDs in applications knowledge of their electronic properties is required.

In this paper, we present an investigation of the electronic structure of $\mathrm{GaSb} / \mathrm{GaAs}$ QDs. We analyze static as well as time-resolved capacitance-voltage $(C-V)$ measurements, in particular deep-level transient measurements (DLTS), in order to determine the key electronic properties, such as the activation energies, the localization energy, and the apparent capture cross sections of the QD ensemble. The analysis of the DLTS signal yields an equivalent to the density of states of the QD ensemble for each individual energy.

\section{Sample}

The sample is grown by MBE. It consists of a layer of $\mathrm{GaSb}$ QDs embedded on the $p$-side of a $p n$ diode. The design allows to charge and discharge the QDs with holes in a controlled way. The layer structure is the following: on top of an $n$-doped GaAs substrate a $300 \mathrm{~nm}$ wide highly doped $\left(n=1 \cdot 10^{18} \mathrm{~cm}^{-3}\right)$ layer is grown as back contact. Then, a $500 \mathrm{~nm}$ wide $p$-doped $\left(p=2 \cdot 10^{16} \mathrm{~cm}^{-3}\right)$ layer is deposited, followed by $7 \mathrm{~nm}$ nominally undoped GaAs. On top of the undoped layer, $3 \mathrm{ML} \mathrm{GaSb}$ are deposited to form QDs with a growth interruption to prevent GaSb ring formation [11]. Details on the growth process can be found in [11, 12]. The QD layer is covered by another $7 \mathrm{~nm}$ of undoped GaAs. 
The rest of the device consists of $700 \mathrm{~nm} p$-doped GaAs $\left(p=2 \cdot 10^{16} \mathrm{~cm}^{-3}\right)$ and a $500 \mathrm{~nm}$ wide highly $p$-doped $(p=$ $1 \cdot 10^{18} \mathrm{~cm}^{-3}$ ) contact layer. The sample is processed into round mesa structures $(r=200 \mu \mathrm{m})$ by standard optical lithography and chemical wet etching techniques. Ohmic top and back contacts are made of Ni/AuGe/Au and Ti/Pt/Au for the $n$-side and $p$-side, respectively, which are annealed for $3 \mathrm{~min}$ at $400^{\circ} \mathrm{C}$.

\section{Theory}

The measurement voltages used in the experiments are in a range where they do not alter the electric field (and hence the band structure) so much that tunneling emission occurs from the QDs. Consequently, only thermal emission is taking place. The thermal emission rate for holes in QDs is then [1315]

$$
e=\gamma T^{2} \sigma_{\infty} \exp \left(-\frac{E_{a}}{k T}\right)
$$

where $\gamma$ is a temperature-independent constant, $T$ the temperature, $\sigma_{\infty}$ the apparent capture cross section, $E_{a}$ the activation energy (emission barrier), and $k$ the Boltzmann constant. The two parameters characteristic for a certain QD ensemble are the activation energy $E_{a}$ and the apparent capture cross section $\sigma_{\infty}$. The activation energy is the barrier which the holes have to overcome during the emission, and the apparent capture cross section is a measure of how well the QDs couple to their surroundings in terms of energy exchange and dissipation [16]. Time-resolved measurements of the hole emission from QDs, especially deep-level transient spectroscopy (DLTS), are used to determine these two parameters.

\section{Results}

For precharacterization, a standard tool to analyze the electronic properties is to make a $C-V$ measurement [14]. A static $C-V$ measurement of the sample at different temperatures is shown in Figure 1. The capacitance is measured between reverse biases of $-0.5 \mathrm{~V}$ and $4 \mathrm{~V}$ at a frequency of $1 \mathrm{MHz}$ and an ac measurement voltage of $100 \mathrm{mV}$. The temperature is increased from $25 \mathrm{~K}$ to $350 \mathrm{~K}$ in steps of $25 \mathrm{~K}$. At low temperature, just the ordinary $1 / \sqrt{V}$-like $C-V$ curve of the diode structure is visible. When the temperature is increased, a plateau-like feature evolves which can be attributed to the hole emission and capture processes of the QDs. In principle, only emission and capture processes which can follow the measurement frequency are visible in the curves. At low temperatures, the emission time constants of the holes in the QDs are too long; hence the holes are not visible. When the thermal energy is increased, the emission rate increases (see (1)). Above a temperature of about $200 \mathrm{~K}$ the upper hole levels contribute to the signal. Eventually, when the thermal energy is sufficient, all hole levels contribute to the signal, and the extension of the plateau saturates, which can be seen for temperatures above $300 \mathrm{~K}$. The second derivative of the $C-V$ curves allows to mark the point where the QDs enter

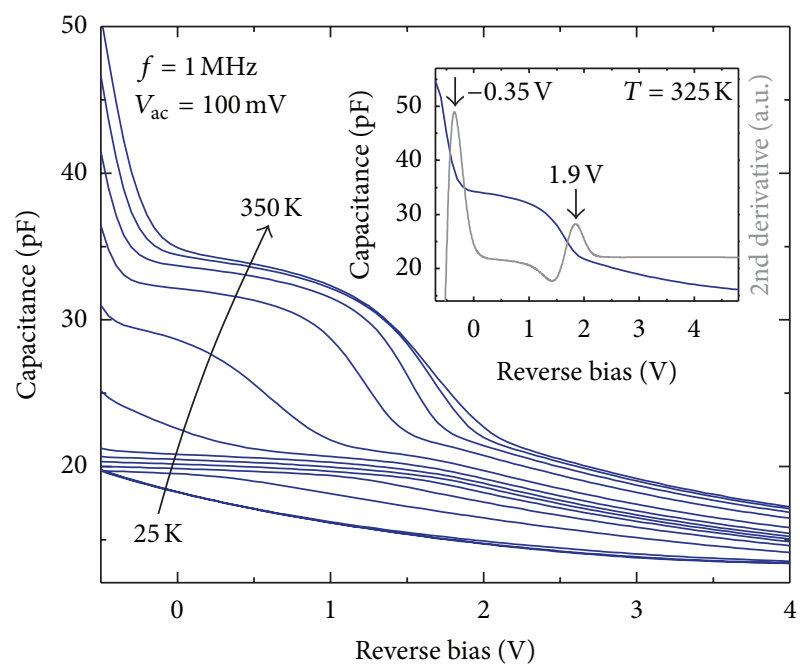

Figure 1: $C-V$ scans for temperatures between $25 \mathrm{~K}$ and $350 \mathrm{~K}$ in steps of $25 \mathrm{~K}$. The evolving plateau due to the emission and capture processes of the QDs is clearly visible. Above $300 \mathrm{~K}$ all hole states in the QD ensemble contribute to the total capacitance. The voltages at which the QDs enter and leave the depletion region can be seen in the second derivative of the $C-V$ curves (see inset).

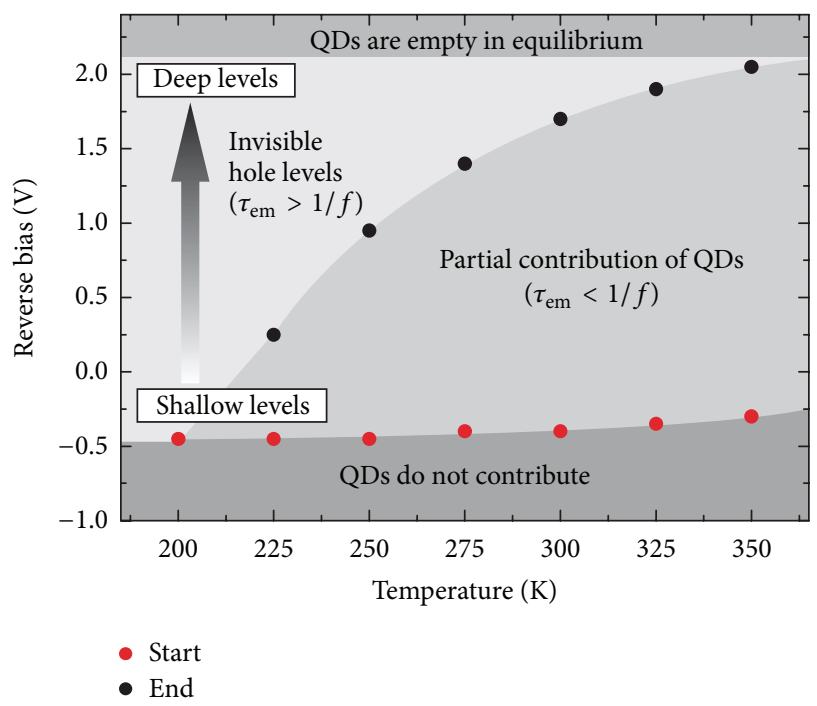

FIgURE 2: Bias voltages of the start and end of the plateau in Figure 1 as a function of temperature (derived from the maxima in the second derivative of the $C-V$ curves; see inset of Figure 1). As the temperature is increased, more and more hole levels are contributing to the total capacitance for a given measurement frequency (here $f=1 \mathrm{MHz}$ ).

and where they leave the depletion region of the pn diode (see inset of Figure 1). The start and end voltages of the $C-V$ curves with a plateau are shown as a function of temperature in Figure 2.

The shaded regions in the graph mark different situations in the diode for a measurement frequency of $1 \mathrm{MHz}$. Below a reverse bias of about $-0.5 \mathrm{~V}$ (i.e., forward direction), the depletion region does not extend to the position of the QD 
layer; hence the QDs are full with holes but cannot be seen in the capacitance. Above a reverse bias of $-0.5 \mathrm{~V}$ the depletion region extends to the QD layer, and at temperatures below the line marked by the end of the plateau (black data points), parts of the hole levels are invisible, as their time constants are too long. At temperatures above the line, the QDs partially contribute to the total capacitance. This means that between a reverse bias of $-0.5 \mathrm{~V}$ and $2 \mathrm{~V}$ the QDs are partly charged in equilibrium. The lower voltages correspond to the shallow hole levels of the dots, and the larger voltages to the deep levels of the QDs. Above a voltage of about 2 V, the QDs are completely discharged in equilibrium. This can also be seen as a steep shoulder of the plateaus in the high-temperature curves in Figure 1. The values for the equilibrium situations are used in the time-resolved $C-V$ measurements to mark the measurement voltages.

The QD ensemble as a whole is then studied by DLTS. Initially, the QDs are completely charged by a voltage pulse to $-0.5 \mathrm{~V}$ (forward direction). Then the emission transient is recorded in the capacitance at a specified measurement voltage $V_{m}$. The emission transients for temperatures between $25 \mathrm{~K}$ and $375 \mathrm{~K}$ are analyzed by the double-boxcar method. The resulting DLTS spectra for three measurement voltages $V_{m}$ and a reference time constant of $\tau_{\text {ref }}=35 \mathrm{~ms}$ are shown in Figure 3. For $V_{m}=1 \mathrm{~V}$, a distinct peak can be seen between $70 \mathrm{~K}$ and $130 \mathrm{~K}$. When the measurement bias is increased to $2 \mathrm{~V}$ and $3 \mathrm{~V}$, the peak broadens on the hightemperature shoulder up to $200 \mathrm{~K}$ and decreases in size. This can be explained by the regions defined in Figure 2. For a measurement bias of $V_{m}=1 \mathrm{~V}$ only the upper hole levels, which correspond to signals at lower temperatures, are emitted. The deeper levels do not contribute to the signal. If the measurement bias is increased to $2 \mathrm{~V}$ and $3 \mathrm{~V}$, these levels are added to the signal and correspond to the signal at higher temperatures. The peak height decreases due to a faster emission from the shallow hole levels, possibly already a result of beginning thermally assisted tunneling processes [17, 18] for the shallow levels at $V_{m}=3 \mathrm{~V}$. The increased emission rate of these levels shifts the signal out of the measurement window (boxcar) and leads to the decreasing signal. The mean activation energy of the QD ensemble is then (at $V_{m}=$ $2 \mathrm{~V}$, where all levels contribute) $115( \pm 5) \mathrm{meV}$ with a mean apparent capture cross section of $\sigma_{\infty}=1 \cdot 10^{-18} \mathrm{~cm}^{2}$.

To study the electronic structure of the QD ensemble in more detail, a charge-selective DLTS method is used [4, 19, 20]. The charging pulse height is much smaller than in the conventional DLTS method, and hence the emission from only a small energy range is probed during the measurement, allowing to observe the emission from very few QD hole levels. The measurement bias $V_{m}$ is changed from $-0.2 \mathrm{~V}$ to $2.2 \mathrm{~V}$ in steps of $0.2 \mathrm{~V}$ while the pulse bias is set to $V_{p}=$ $V_{m}-0.2 \mathrm{~V}$. The resulting DLTS spectra are shown in Figure 4 for a reference time constant of $\tau_{\text {ref }}=35 \mathrm{~ms}$. A peak structure can be seen which shifts from $90 \mathrm{~K}$ for $V_{m}=-0.2 \mathrm{~V}$ to about $210 \mathrm{~K}$ for $V_{m}=2.2 \mathrm{~V}$. The peak height is decreasing for increasing measurement bias. From the DLTS spectra, Arrhenius plots (not shown) can be derived, which can be analyzed by fitting the emission rate equation (1) to the data, yielding the activation energy $E_{a}$ and the apparent capture

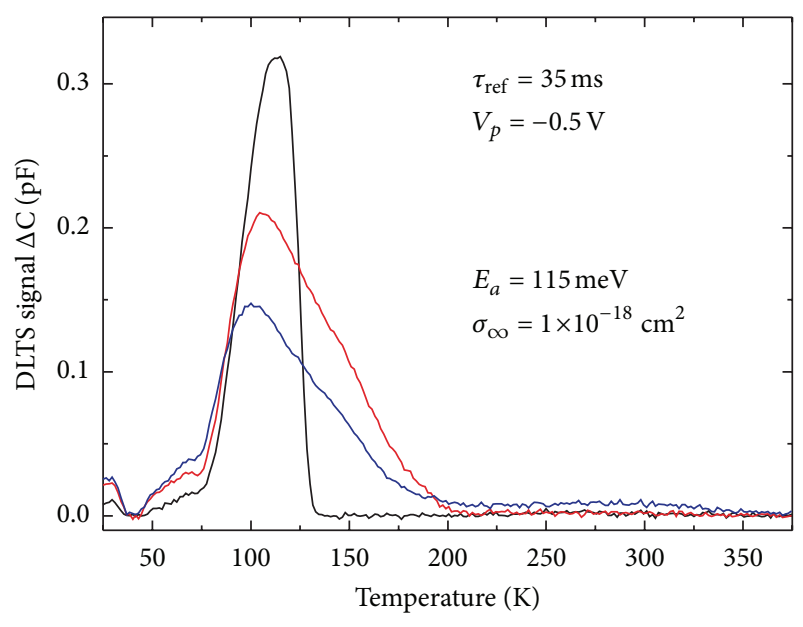

$$
\begin{aligned}
& V_{m}= \\
& -1 \mathrm{~V} \\
& -\quad 2 \mathrm{~V} \\
& -3 \mathrm{~V}
\end{aligned}
$$

FIGURE 3: Conventional DLTS measurement for different measurement voltages $V_{m}$ and a reference time constant of $\tau_{\text {ref }}=35 \mathrm{~ms}$. The activation energy corresponds to the measurement at $V_{m}=2 \mathrm{~V}$.

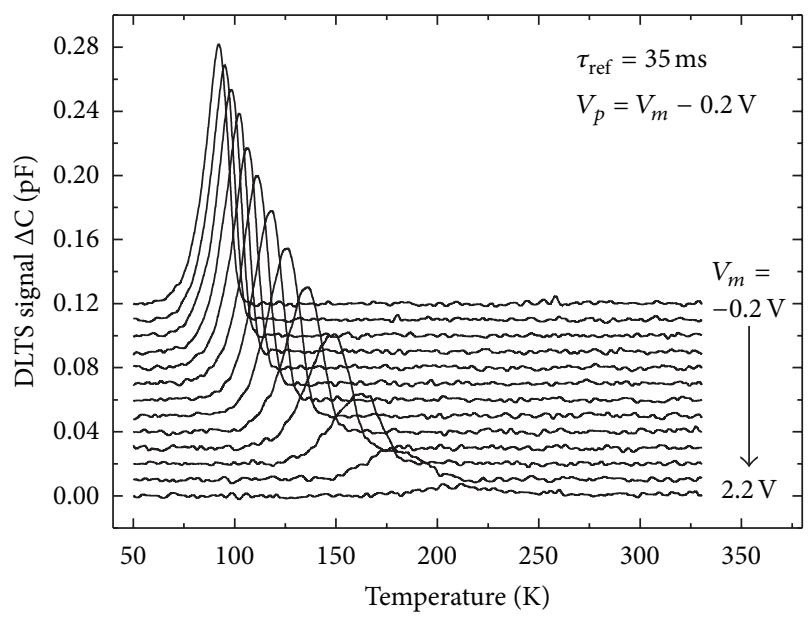

FIGURE 4: Charge-selective DLTS spectra for measurement voltages $V_{m}$ between $-0.2 \mathrm{~V}$ (forward direction) and $2.2 \mathrm{~V}$ (reverse direction) and a reference time constant of $\tau_{\text {ref }}=35 \mathrm{~ms}$. The charging pulse amplitude is fixed to $0.2 \mathrm{~V}$. The peaks for the deep hole states shift toward higher temperatures and decrease in height.

cross section $\sigma_{\infty}$ as fit parameters. The activation energies and corresponding apparent capture cross sections for the individual measurement voltages are shown in Figure 5. The activation energy $E_{a}$ increases for increasing reverse bias from $90 \mathrm{meV}$ at $V_{m}=-0.2 \mathrm{~V}$ to about $330 \mathrm{meV}$ at $V_{m}=2.2 \mathrm{~V}$, indicating that with increasing measurement bias deeper hole levels of the QD ensemble are probed. As the DLTS signal vanishes completely above a bias of $2.2 \mathrm{~V}$, the maximum activation energy belongs to the localization energy of the QD ensemble. The apparent capture cross sections show a similar trend to that of the activation energies. They increase from 


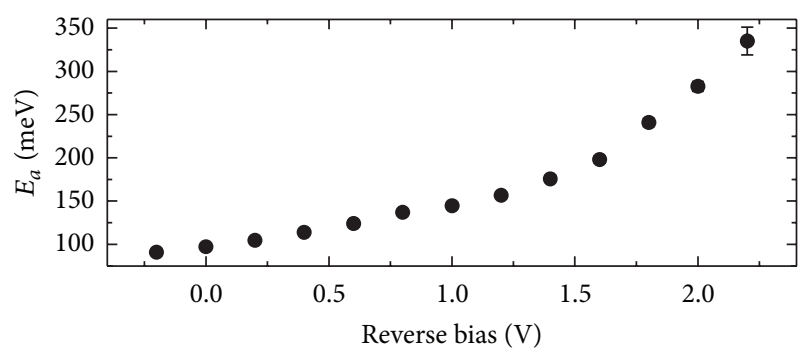

(a)

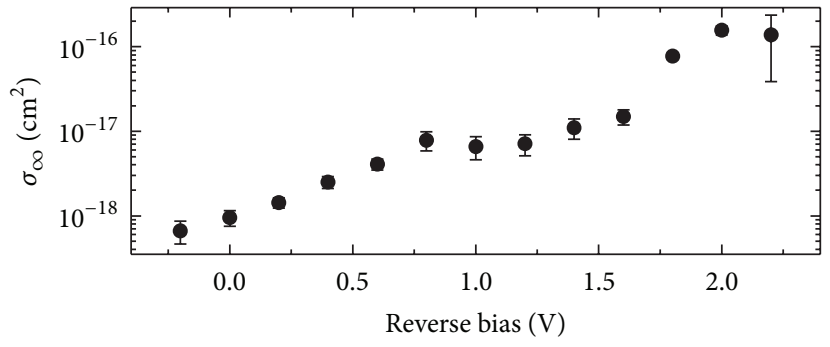

(b)

Figure 5: (a) Activation energies and (b) apparent capture cross sections for holes derived from the charge-selective DLTS spectra.

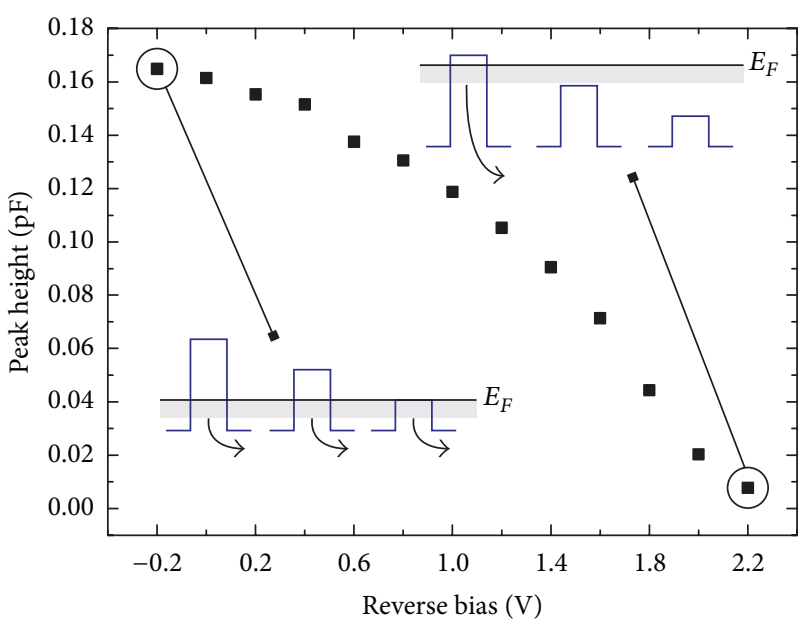

FIGURE 6: Peak heights as function of measurement voltage $V_{m}$. The insets schematically show the contribution of QDs with different individual localization energies. The shaded areas indicate the energy range that is probed during a single measurement step. The localization energy of each individual QD depends on the dot size and its composition which have not been indicated in the insets separately.

about $10^{-18} \mathrm{~cm}^{2}$ at $V_{m}=-0.2 \mathrm{~V}$ to about $10^{-16} \mathrm{~cm}^{2}$ at $V_{m}=$ $2.2 \mathrm{~V}$. The localization energy is then $335( \pm 15) \mathrm{meV}$ with a corresponding apparent capture cross section of $1 \cdot 10^{-16} \mathrm{~cm}^{2}$ with an error of about half an order of magnitude.

The peak height of each individual DLTS peak versus the measurement bias is shown in Figure 6. The peak heights decrease due to a decreasing number of hole states in the density of states (DOS) of the QD ensemble, which is illustrated in the insets of Figure 6. For a small measurement bias, all sizes of QDs contribute to the signal, the larger with their shallow levels, and the smaller with their deep levels. In contrast, when the measurement bias is increased, the smaller QDs are always empty throughout the measurement cycle, and hence do not contribute to the signal. Only the deepest levels of the large QDs are charged and discharged during the pulse. Hence, the localization energy measured belongs to the largest confinement energies in the QD ensemble, which can be attributed to dots with large sizes and/or large GaSb content.

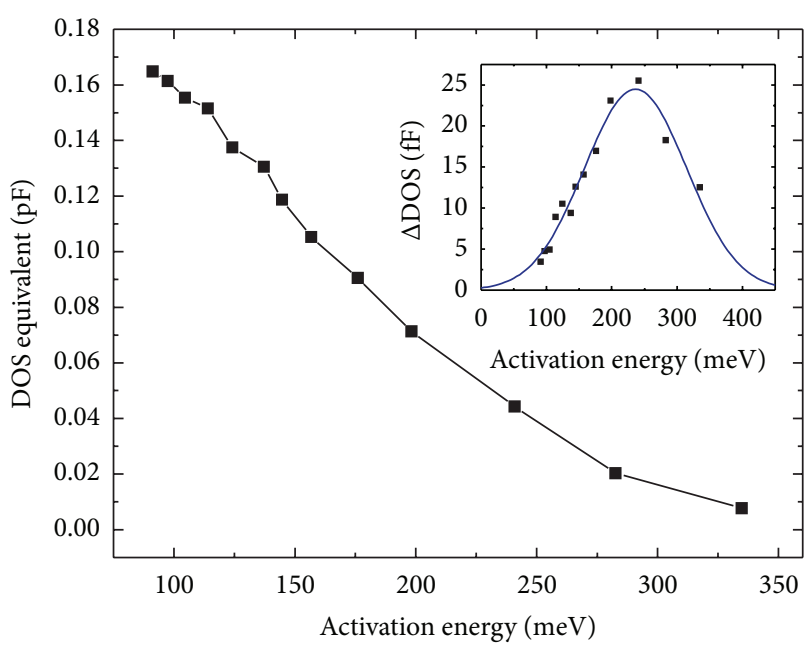

FIGURE 7: Density of states equivalent as a function of the activation energies determined from the charge-selective DLTS. The inset shows the individual contribution of each additional data point to the DOS equivalent; the curve resembles the density function of the confinement energies of the QDs in the ensemble.

The peak heights can also be plotted versus the activation energies that were measured at the respective measurement voltage (Figure 5(a)). The resulting curve is shown in Figure 7 and resembles an equivalent to the density of states of the QD ensemble, indicating a value proportional to the number of hole states at a certain energetic depth of the QD ensemble. When the additional contribution of each data point to the DOS equivalent is plotted versus the activation energy (see inset of Figure 7), the density function of the confinement energies of the QDs in the ensemble is obtained. The mean value of that function is located around an activation energy of $\sim 240 \mathrm{meV}$.

\section{Discussion}

The $C-V$ measurements and the conventional and chargeselective DLTS measurements have yielded the activation energies, the apparent capture cross sections, the localization energy of the QD ensemble, and a curve equivalent to the density of states between a potential depth of 90 and $340 \mathrm{meV}$ in the QD ensemble. When comparing the measured values 
with previous measurements in the same material system $[4,19,21]$, the localization energy is much smaller than in all the other samples, except for a single one which has a comparable potential depth [22]. It can be concluded from the previous measurements [4] and 8-band- $k \cdot p$-calculations [21] that the QDs are very small and/or have a small GaSb content. Also, the apparent capture cross sections around values of $10^{-16} \mathrm{~cm}^{2}$ are unusually small compared to previous studies where values between $10^{-13} \mathrm{~cm}^{2}$ and $10^{-11} \mathrm{~cm}^{2}$ were found [4]. The density of states equivalent curve resembles part of the distribution function that one would expect if the density function of the confinement energies of the individual QDs in the ensemble is assumed to have a Gaussian shape, which is plausible and is also confirmed by the data.

\section{Summary}

We have studied the electronic properties of $\mathrm{GaSb} / \mathrm{GaAs}$ QDs by $C-V$ spectroscopy and DLTS. The $C-V$ measurements revealed details about the charging state of the QDs with respect to the applied bias. The QD ensemble has a mean activation energy of $115( \pm 5) \mathrm{meV}$ and a localization energy of $335( \pm 15) \mathrm{meV}$ with a corresponding apparent capture cross section of $1 \cdot 10^{-16} \mathrm{~cm}^{2}$ with an error of about half an order of magnitude. The localization energy belongs to the QDs with the largest activation energies, which are found at the upper edge of the Gaussian size distribution.

\section{Acknowledgments}

The authors gratefully acknowledge financial support by the EC NanoSci-E+ Project QD2D (BI284/30-1), DFG Contract no. BI284/29-1, and the BMBF Project HOFUS. The work of part of the authors was funded by the Deanship of Scientific Research (DSR), King Abdulaziz University, under Grant no. 2-4-1432/HiCi. These authors, therefore, acknowledge with thanks DSR technical and financial support.

\section{References}

[1] M. Hayne, J. Maes, S. Bersier et al., "Electron localization by selfassembled GaSb/GaAs quantum dots," Applied Physics Letters, vol. 82, no. 24, pp. 4355-4357, 2003.

[2] A. Marent, T. Nowozin, M. Geller, and D. Bimberg, “The QDflash: a quantum dot-based memory device," Semiconductor Science and Technology, vol. 26, no. 1, Article ID 014026, 2011.

[3] T. Nowozin, A. Marent, G. Hönig et al., “Time-resolved hightemperature detection with single charge resolution of holes tunneling into many-particle quantum dot states," Physical Review B, vol. 84, no. 7, Article ID 075309, 7 pages, 2011.

[4] T. Nowozin, L. Bonato, A. Högner et al., " $800 \mathrm{meV}$ localization energy in $\mathrm{GaSb} / \mathrm{GaAs} / \mathrm{Al}_{0.3} \mathrm{Ga}_{0.7}$ As quantum dots," Applied Physics Letters, vol. 102, no. 5, Article ID 052115, 4 pages, 2013.

[5] T. Nowozin, D. Bimberg, K. Daqrouq, M. N. Ajour, and M. Awedh, "Materials for future quantum dot-based memories," Journal of Nanomaterials, vol. 2013, Article ID 215613, 6 pages, 2013.
[6] C. K. Sun, G. Wang, J. E. Bowers et al., "Optical investigations of the dynamic behavior of GaSb/GaAs quantum dots," Applied Physics Letters, vol. 68, no. 11, pp. 1543-1545, 1996.

[7] K. Gradkowski, N. Pavarelli, T. J. Ochalski et al., "Complex emission dynamics of type-II GaSb/GaAs quantum dots," Applied Physics Letters, vol. 95, no. 6, Article ID 061102, 3 pages, 2009.

[8] R. B. Laghumavarapu, A. Moscho, A. Khoshakhlagh, M. ElEmawy, L. F. Lester, and D. L. Huffaker, "GaSbGaAs type II quantum dot solar cells for enhanced infrared spectral response," Applied Physics Letters, vol. 90, no. 17, Article ID 173125, 3 pages, 2007.

[9] B. M. Kinder and E. M. Goldys, "Microstructural evolution of GaSb self-assembled islands grown by metalorganic chemical vapor deposition," Applied Physics Letters, vol. 73, no. 9, article 1233, 3 pages, 1998.

[10] L. Müller-Kirsch, R. Heitz, U. W. Pohl et al., "Temporal evolution of $\mathrm{GaSb} / \mathrm{GaAs}$ quantum dot formation," Applied Physics Letters, vol. 79, no. 7, article 1027, 3 pages, 2001.

[11] C. C. Tseng, S. C. Mai, W. H. Lin et al., "Influence of as on the morphologies and optical characteristics of $\mathrm{GaSb} / \mathrm{GaAs}$ quantum dots," IEEE Journal of Quantum Electronics, vol. 47, no. 3, pp. 335-339, 2011.

[12] S. Y. Lin, C. C. Tseng, W. H. Lin et al., "Room-temperature operation type-II GaSb/GaAs quantum-dot infrared light-emitting diode," Applied Physics Letters, vol. 96, no. 12, Article ID 123503, 3 pages, 2010.

[13] D. V. Lang, "Deep-level transient spectroscopy: a new method to characterize traps in semiconductors," Journal of Applied Physics, vol. 45, no. 7, article 3023, 10 pages, 1974.

[14] P. Blood and J. W. Orton, The Electrical Characterization of Semiconductors: Majority Carriers and Electron States, Academic Press, London, UK, 1992.

[15] C. M. A. Kapteyn, M. Lion, R. Heitz et al., "Hole and electron emission from InAs quantum dots," Applied Physics Letters, vol. 76, no. 12, pp. 1573-1575, 2000.

[16] J. Bourgoin and M. Lannoo, Point Defects in Semiconductors II-Experimental Aspects, vol. 35 of Springer Series in Solid-State Sciences, Springer, Berlin, Germany, 1983.

[17] G. Vincent, A. Chantre, and D. Bois, "Electric field effect on the thermal emission of traps in semiconductor junctions," Journal of Applied Physics, vol. 50, no. 8, pp. 5484-5487, 1979.

[18] T. Nowozin, A. Marent, M. Geller, D. Bimberg, N. Akçay, and N. Öncan, "Temperature and electric field dependence of the carrier emission processes in a quantum dot-based memory structure," Applied Physics Letters, vol. 94, no. 4, Article ID 042108, 3 pages, 2009.

[19] M. Geller, C. Kapteyn, L. Müller-Kirsch, R. Heitz, and D. Bimberg, " $450 \mathrm{meV}$ hole localization in $\mathrm{GaSb} / \mathrm{GaAs}$ quantum dots," Applied Physics Letters, vol. 82, no. 16, pp. 2706-2708, 2003.

[20] A. Marent, M. Geller, A. Schliwa et al., " $10^{6}$ years extrapolated hole storage time in GaSb/AlAs quantum dots," Applied Physics Letters, vol. 91, no. 24, Article ID 242109, 3 pages, 2007.

[21] T. Nowozin, A. Marent, L. Bonato et al., "Linking structural and electronic properties of high-purity self-assembled $\mathrm{GaSb} / \mathrm{GaAs}$ quantum dots," Physical Review B, vol. 86, no. 3, Article ID 035305, 6 pages, 2012.

[22] J. Hwang, A. J. Martin, J. M. Millunchick, and J. D. Phillips, "Thermal emission in type-II GaSb/GaAs quantum dots and prospects for intermediate band solar energy conversion," Journal of Applied Physics, vol. 111, no. 7, Article ID 074514, 5 pages, 2012. 

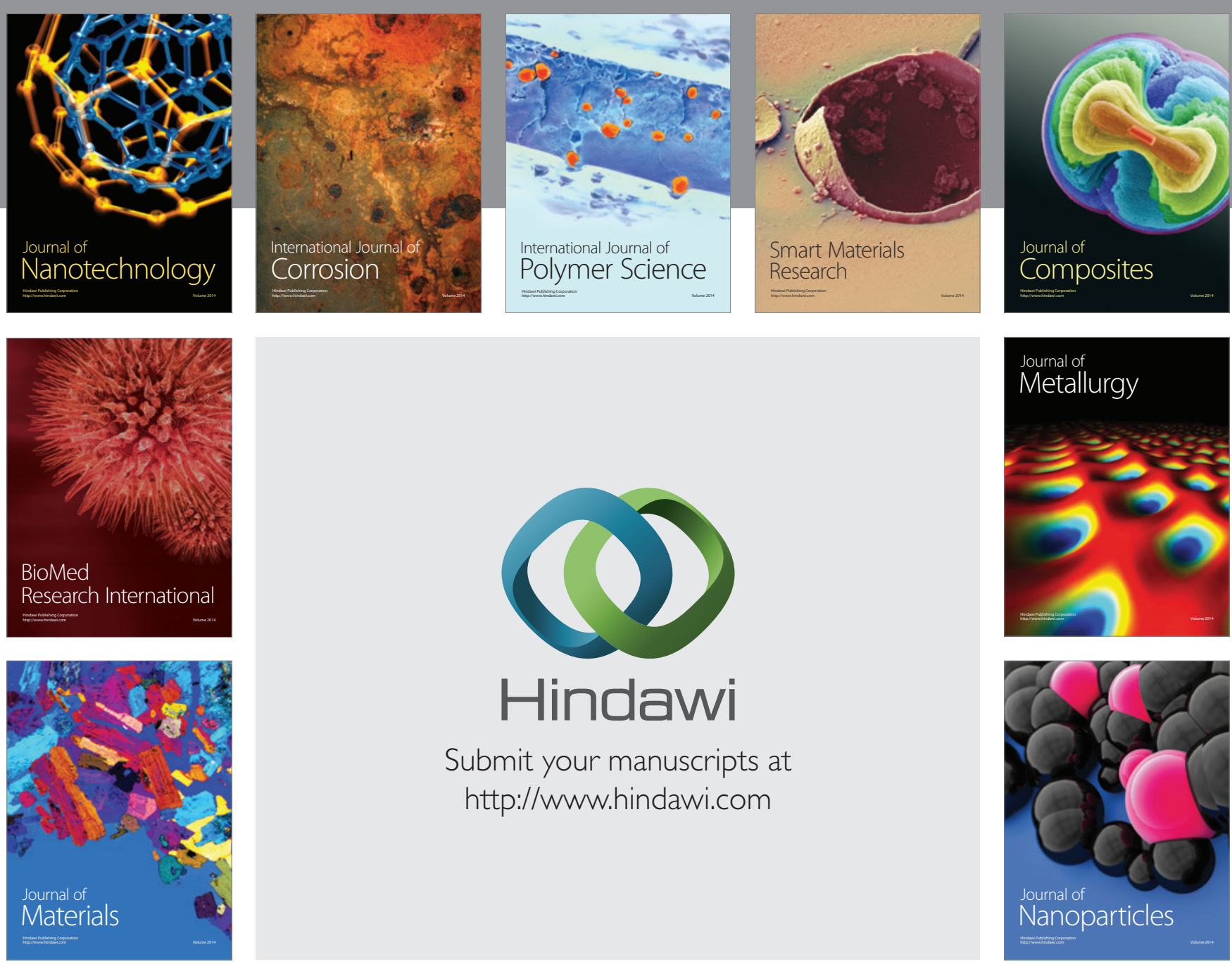

Submit your manuscripts at http://www.hindawi.com
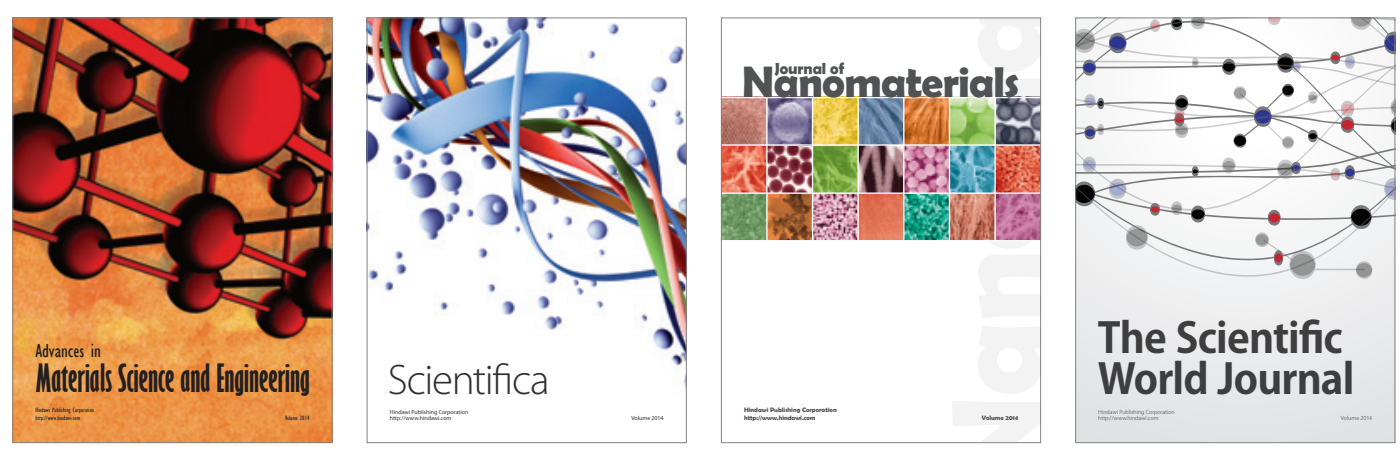

\section{The Scientific World Journal}
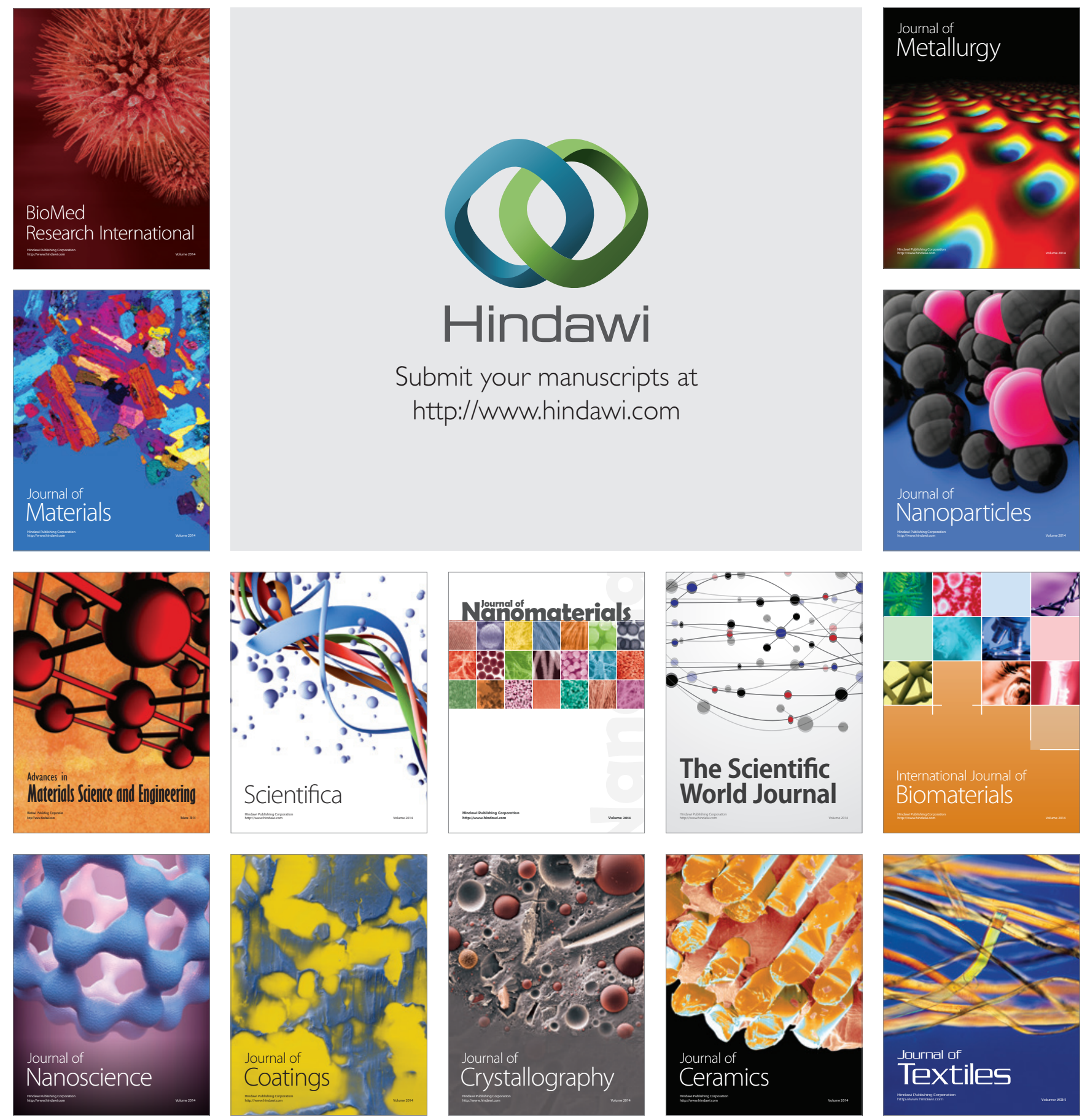